\title{
Outpatient Management of Bronchial Asthma: A Comparative Analysis Between Guideline-Directed Management and Usual Management
}

\author{
Jeyanthan Jayakumaran ${ }^{\mathrm{a}, \mathrm{d}}$, Krystal Hunter ${ }^{\mathrm{b}}$, Satyajeet Roy ${ }^{\mathrm{a}, \mathrm{c}}$
}

\begin{abstract}
Background: Bronchial asthma is a common controllable disease that causes a serious economic and social burden. The Global Initiative for Asthma (GINA) was developed to help guide clinicians in appropriate management of asthma. Despite the existence of published guidelines, common practice in many primary care clinics follows usual care based on clinical gestalt. This study aims to determine if there is a statistically significant difference in outcomes between patients receiving guideline-directed therapy when compared to those receiving usual clinician therapy.
\end{abstract}

Methods: A total of 300 patients were included in this study. Among them, 139 patients received guideline-directed medical therapy (GDMT group) and 161 received usual medical therapy (UMT group). Logistic regression models were utilized to determine if there was a significant difference in outcomes for patients comparing number of exacerbations and number of hospitalizations.

Results: More patients in GDMT group suffered from recorded exacerbations in the prior year with $43.9 \%$ having one, $3.6 \%$ having two, and $0.7 \%$ having three, compared to the frequencies of exacerbations in the UMT group $(29.2 \%, 1.9 \%$, and $1.2 \%$, respectively $)(\mathrm{P}<0.05)$. Cumulative number of hospitalizations due to asthma exacerbations in the prior year was also higher in GDMT group compared to the UMT group (one in 5.8\% GDMT vs. 3.1\% UMT; two in 0.0\% GDMT vs. $0.6 \% \mathrm{UMT})$ without statistically significant difference $(\mathrm{P}=0.349)$.

Conclusions: Primary care providers' adherence to the 2018 GINA guidelines for asthma treatment did not offer benefit to patient outcomes, such as number of exacerbations or hospitalizations, compared to the usual medical care of bronchial asthma. Patient-tailored

Manuscript submitted May 10, 2020, accepted May 25, 2020

Published online June 4, 2020

aDepartment of Medicine, Cooper University Health Care, Camden, NJ, USA ${ }^{b}$ Cooper Research Institute, Cooper Medical School of Rowan University, Camden, NJ, USA

${ }^{\mathrm{c} C o o p e r}$ Medical School of Rowan University, Camden, NJ, USA

${ }^{\mathrm{d} C o r r e s p o n d i n g ~ A u t h o r: ~ J e y a n t h a n ~ J a y a k u m a r a n, ~ D e p a r t m e n t ~ o f ~ M e d i c i n e, ~}$ Cooper University Health Care, 1103 North Kings Highway, Suite 203, Cherry Hill, NJ, USA. Email: Jayakumaran-Jeyanthan@cooperhealth.edu

doi: https://doi.org/10.14740/jocmr4208 care may offer reduction in the rates of exacerbations and hospitalization.

Keywords: Bronchial asthma; Management of asthma; Guidelinedirected management; Usual management

\section{Introduction}

Bronchial asthma is a common controllable disease that causes a serious economic and social burden. Currently the disease is recognized by the World Health Organization as the most common chronic inflammatory disease characterized by paroxysmal symptomatic periods [1]. Asthma is overwhelmingly present in both the adult (5-7\%) and pediatric (10-12\%) populations [2]. In the USA specifically, asthma prevalence has steadily risen since the 1980s [3], as does its cost on the US health care system. From 2008 to 2013, the annual economic burden of asthma was estimated at more than $\$ 81.9$ billion, with an annual per-person incremental medical cost of $\$ 3,266$ [4]. Many factors related to a person's environment have been attributed to the increase in asthma prevalence, including indoor chemical use, external pollution, and rapid urbanization as well as its associated negative consequences [5]. Urbanization and the loss of protective factors have led to the synthesis of the hygiene hypothesis as well, which aggresses that over the last 30 years there is an observed inverse relationship in the prevalence of infectious diseases and the frequency of allergic and autoimmune disease processes [6].

The diagnosis of bronchial asthma requires diagnostic workup in patients who present with dry cough especially at night, rhonchi, wheezing, chest tightness, and shortness of breath, etc. The diagnostic workup includes pulmonary function testing with or without methacholine challenge.

If it is thought to be secondary to an allergic process, skin testing and immunoglobulin E (IgE) levels can also be tested [7]. Once the diagnosis is made, patients then require close follow-up and appropriate therapy based on stepwise guidelines.

The Global Initiative for Asthma (GINA) was developed to help guide clinicians in appropriate management of asthma, and has been updated most recently in 2018 [8]. In contrast to the Global Initiative for Chronic Obstructive Lung Disease (GOLD) criteria for chronic obstructive pulmonary disease 
(COPD), which combines objective evidence in the form of pulmonary function testing and symptoms, the severity of asthma as outlined by GINA is based on patient symptomatology. Asthma severity is graded based on number of daytime symptoms in a week, nocturnal awakenings due to asthma, how often rescue inhaler use is required, and any limitations of daily activity secondary to asthma. Treatment follows a step-up/step-down model in order to properly control patients' symptoms [8]. Therapeutic agents shown to be affective are those that decrease bronchospasm, such as short- and long-acting beta agonists as well as those that decrease inflammation such as inhaled corticosteroids (ICSs) [9].

The GINA guideline-directed therapy is seen as the standard of care for outpatient management of bronchial asthma and includes utilizing an as needed short-acting inhaled betaagonist (SABA) at step 1, adding on a standing ICS at step 2 , utilizing mid-dose ICS/long-acting inhaled beta-agonist (LABA) at step 3, and increasing doses of ICS/LABA at step 4 , with step 5 being referral to expert for appropriate add-on therapy. Step-up guidelines do come with the caveat that patients should be continually evaluated at every outpatient visit for possible step-down therapy [8]. Stepping down and deescalating agents help avoid adverse effects related to longterm use of medications, as well as cost for medications for the patients [10].

Adverse outcomes of poor asthma control can result in an increase in asthma exacerbations which in turn are a major cause of disease morbidity, increases in health care costs, and in a subset of patients, acceleration of disease progression [11]. Asthma exacerbations continue to be a major financial burden to patients and society, with one 2007 study estimating that patients with exacerbations incurred over double the costs when compared to patients without exacerbations [12]. Past studies have found that patients requiring an emergency room visit or hospitalization for an asthma exacerbation are at significantly increased risk for future exacerbations [13]. Despite the existence of published guidelines, common practice in many primary care clinics follows usual care based on clinical gestalt. We performed a retrospective chart review of our single outpatient office with aims to determine if there is an appreciable difference in the outcomes between patients receiving guidelinedirected therapy and those receiving usual clinician therapy.

\section{Materials and Methods}

\section{Study selection}

This study was a retrospective electronic medical record (EMR) review observing the management of patients with asthma in the primary care setting who were seen from July 1 , 2018 to June 30, 2019. The inclusion criteria were patients of age 18 years and older who were seen in the outpatient office with a documented diagnosis of asthma. The exclusion criteria were patients under the age of 18 year, or those who did not have the diagnosis of asthma. This study was reviewed and approved by the Institutional Review Board at Cooper University Health Care, Camden, NJ, USA; and this study was fully compliant with the ethical standards set forth by the Cooper University Health Care Institutional Review Board.

\section{Data collection}

For each patient, pertinent data were collected including: age, gender, race, body mass index (BMI), smoking history, presence of comorbid conditions including allergic rhinitis, atopic dermatitis, nasal polyps, eosinophilia, congestive heart failure, COPD, end-stage renal disease (ESRD), end-stage liver disease (ESLD), anemia, rheumatoid arthritis, multisystem disease, psychiatric diagnoses, and gastroesophageal reflux disease (GERD). The following data related to asthma were collected: severity of disease, number of exacerbations in last year (defined as clinically significant dyspnea and wheezing requiring outpatient provider evaluation), number of hospitalizations in last year, adherence to guideline-driven medical therapy, documented medication use, such as SABA, ICS, LABA, long-acting anti-muscarinic agent (LAMA), leukotriene inhibitor, theophylline, zileuton, and omalizumab.

\section{Statistical analysis}

Data were collected and stored in a Microsoft Excel (2016, Redmond, WA, USA) spreadsheet. The two study groups compared were defined as patients receiving guideline-directed medical therapy (GDMT) group and those that received usual medical therapy (UMT) group delivered by their primary care physicians. Statistical analysis was done by employing SPSS (Statistical Package for the Social Sciences, version 15.01, IBM, Armonk, NY, USA). Independent $t$-tests were utilized to compare continuous variables between the study groups. Chi-square tests were utilized to compare categorical variables between the study groups. Logistic regression models were utilized to determine if there was a significant difference in outcomes for patients comparing number of exacerbations and number of hospitalizations. A P value of $<0.05$ was utilized to determine statistical significance.

\section{Results}

A total of 300 patients were included in this study, with 139 in the GDMT group and 161 in the UMT group. The mean age of patients in the GDMT group was $56.67 \pm 15.57$ year compared to $56.70 \pm 15.79$ year in the UMT group $(\mathrm{P}=0.986)$ (Table 1$)$. The majority of patients were female in both the groups with $71.2 \%$ in the GDMT group and $72.0 \%$ in the UMT group. The patients in either group were predominantly white with $63.3 \%$ in the GDMT group and $66.5 \%$ in the UMT group, followed by black $($ GDMT $=17.3 \%, \mathrm{UMT}=13.7 \%)$ and Hispanic $($ GDMT $=15.1 \%$, UMT $=14.3 \%)$. The two most common history findings in either group were positive cigarette smoking history $(\mathrm{GDMT}=37.4 \%, \mathrm{UMT}=44.1 \%)$ and history of allergic rhinitis $(\mathrm{GDMT}=37.4 \%$, UMT $=39.1 \%)($ Tables 1,2$)$. The differences in the frequencies were not statistically significant. 
Table 1. Demographics and Clinical Characteristics

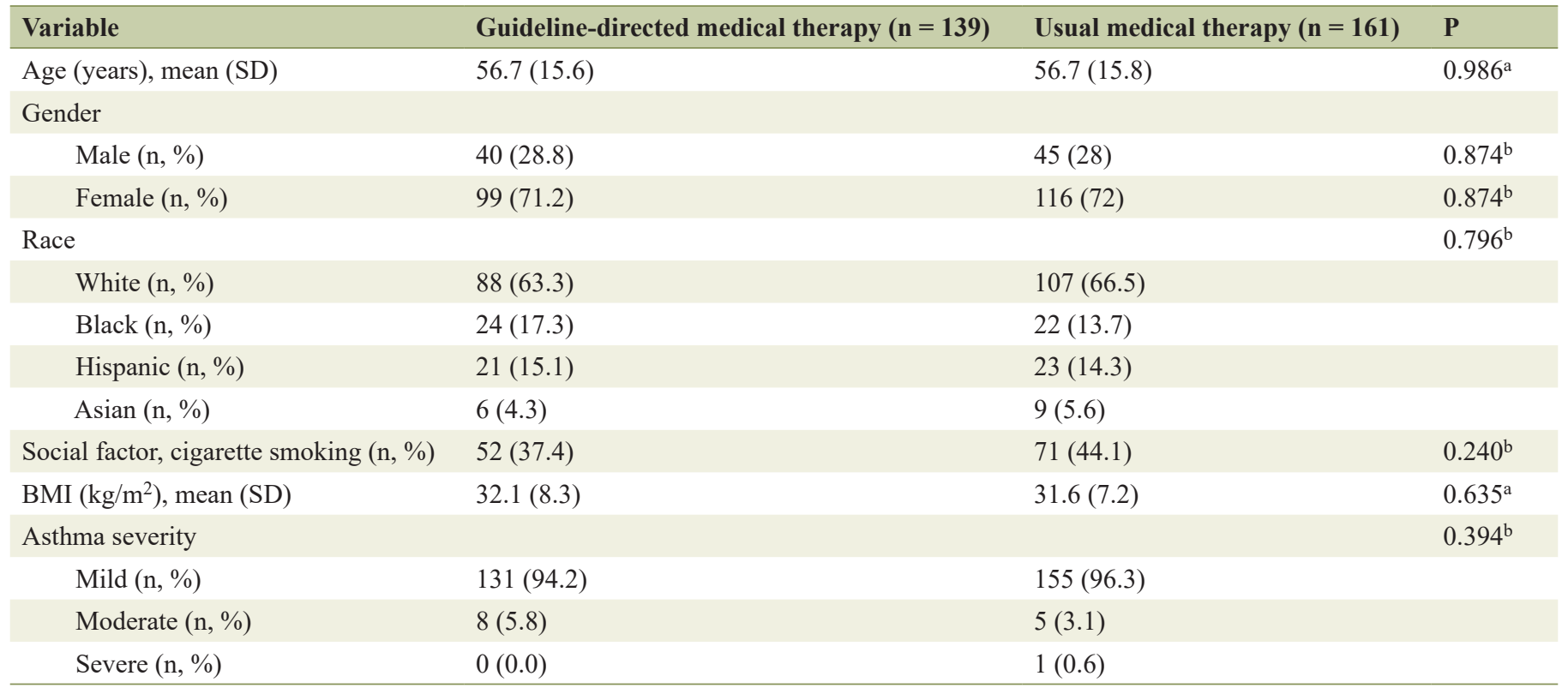

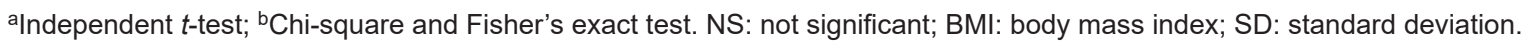

Among the various comorbidities, patients being treated for asthma in our clinics most commonly suffered from psychiatric diagnoses (GDMT $=36.0 \%, \mathrm{UMT}=50.9 \%)$ and GERD $(\mathrm{GDMT}=43.9 \%$, UMT $=46.6 \%)($ Table 2$)$. Logistic regression analysis showed that the patients suffering from congestive heart failure appear to have increased odds of suffering from exacerbations. All other associated medical conditions did not have a significant association with the rate of exacerbation. Patients with moderate to severe asthma had on average 9.7 times greater odds of having exacerbations when compared to those with mild asthma. In addition, patients with allergic rhinitis were found to have two times greater odds of having exacerbations (Table 3).

The majority of patient's in the GDMT group were treated with an SABA with $99.3 \%$ receiving this medication, compared to only $51.6 \%$ of those in the UMT group. The difference was statistically significant $(\mathrm{P}<0.05)$ (Table 4). ICS was the next most commonly prescribed treatment in the GDMT group with $49.6 \%$ of patients receiving this medication. Out of these patients $21.7 \%$ were prescribed a low dosage, $39.1 \%$ were prescribed a medium dosage, and $39.1 \%$ were prescribed high dosage. This was significantly higher than the frequency

Table 2. Associated Comorbid Conditions

\begin{tabular}{|c|c|c|c|}
\hline Variable & Guideline-directed medical therapy $(\mathrm{n}=139)$ & Usual medical therapy $(n=161)$ & $\mathbf{P}^{\mathbf{a}}$ \\
\hline CHF (n, \%) & $14(10.1)$ & $16(9.9)$ & 0.969 \\
\hline $\operatorname{ESRD}(n, \%)$ & $1(0.7)$ & $0(0.0)$ & 0.463 \\
\hline Anemia $(\mathrm{n}, \%)$ & $22(15.8)$ & $27(16.8)$ & 0.826 \\
\hline Rheumatoid arthritis (n, \%) & $5(3.6)$ & $10(6.2)$ & 0.300 \\
\hline Psychiatric diseases (n, \%) & $50(36.0)$ & $82(50.9)$ & 0.650 \\
\hline GERD (n, \%) & $61(43.9)$ & $75(46.6)$ & 0.640 \\
\hline COPD (n, \%) & $11(7.9)$ & $19(11.8)$ & 0.263 \\
\hline Allergic rhinitis (n, \%) & $52(5.8)$ & $63(39.1)$ & 0.760 \\
\hline Atopic dermatitis (n, \%) & $8(17.6)$ & $14(8.7)$ & 0.330 \\
\hline
\end{tabular}

${ }^{a}$ Chi-square and Fisher's exact test. CHF: congestive heart failure; ESRD: end-stage renal disease; ESLD: end-stage liver disease; GERD: gastroesophageal reflux disorder; COPD: chronic obstructive pulmonary disease. 
Table 3. Comorbidities and Relation to Exacerbations

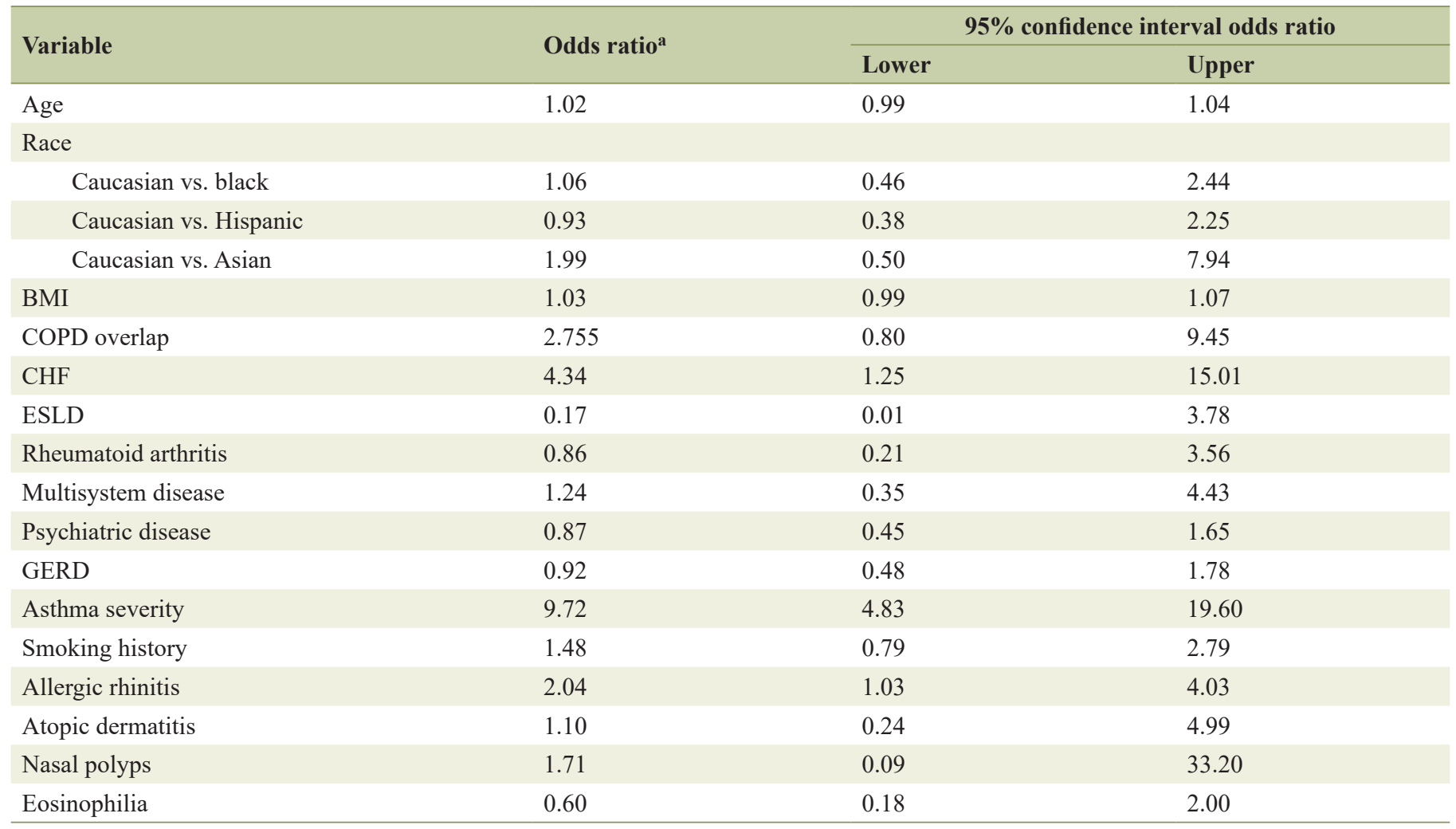

aLogistic regression. BMI: body mass index; COPD: chronic obstructive pulmonary disease; CHF: congestive heart failure; ESLD: end-stage liver disease; GERD: gastroesophageal reflux disorder.

of ICS prescription to patients in the UMT group at 33.5\% (P< 0.05) (Table 4). Among the UMT group patients, 35.2\% were prescribed a low dosage, $40.7 \%$ were prescribed a medium dosage, and $24.1 \%$ were prescribed a high dosage. Patients were also prescribed LABA at a significantly higher rate in the GDMT group compared to the UMT group (34.5\% vs. $24.1 \%$ ) $(\mathrm{P}<0.05)$ (Table 4). Of note, both LAMA and leukotriene inhibitors were prescribed at a significantly higher frequencies in the UMT group ( $9.9 \%$ and $37.9 \%$, respectively) when com- pared to the GDMT group (2.2\% and $16.5 \%$, respectively) (P $<0.05$ ) (Table 4). Prescription of omalizumab was rare and only seen in $1.4 \%$ of the GDMT group and none in the UMT group (Table 4).

When comparing outcomes between the two groups, we found that significantly more patients in the GDMT group suffered from recorded exacerbations with $43.9 \%$ having one, $3.6 \%$ having two, and $0.7 \%$ having three recorded exacerbations in the prior year, compared to the frequencies of exac-

Table 4. Medications Prescribed

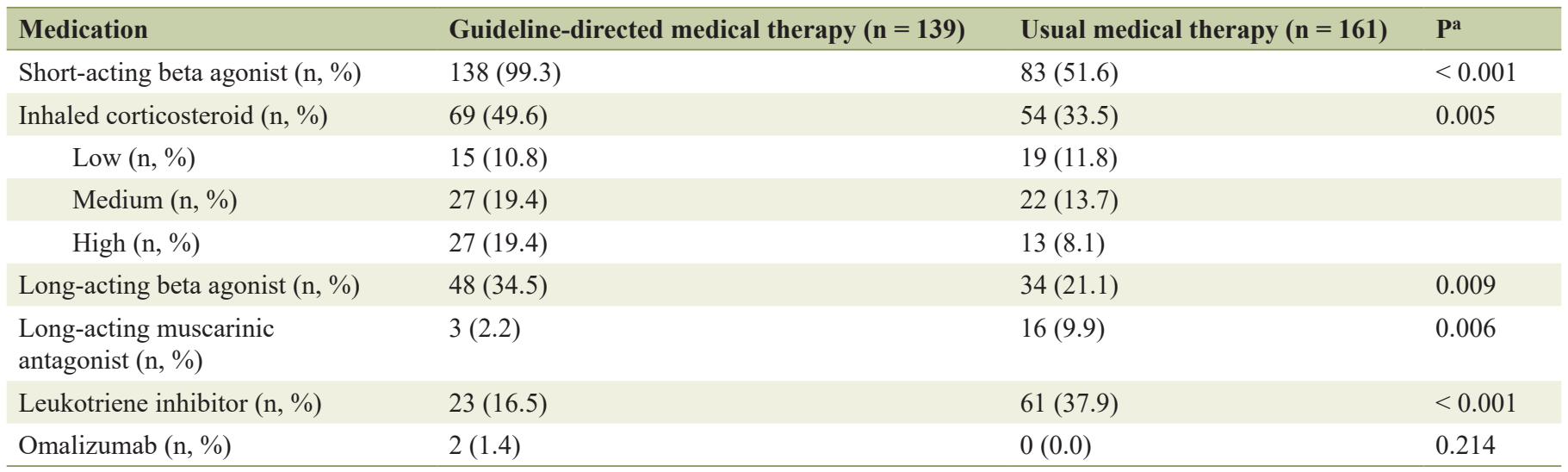

aChi-square and Fisher's exact test. 
Table 5. Exacerbations and Hospitalizations

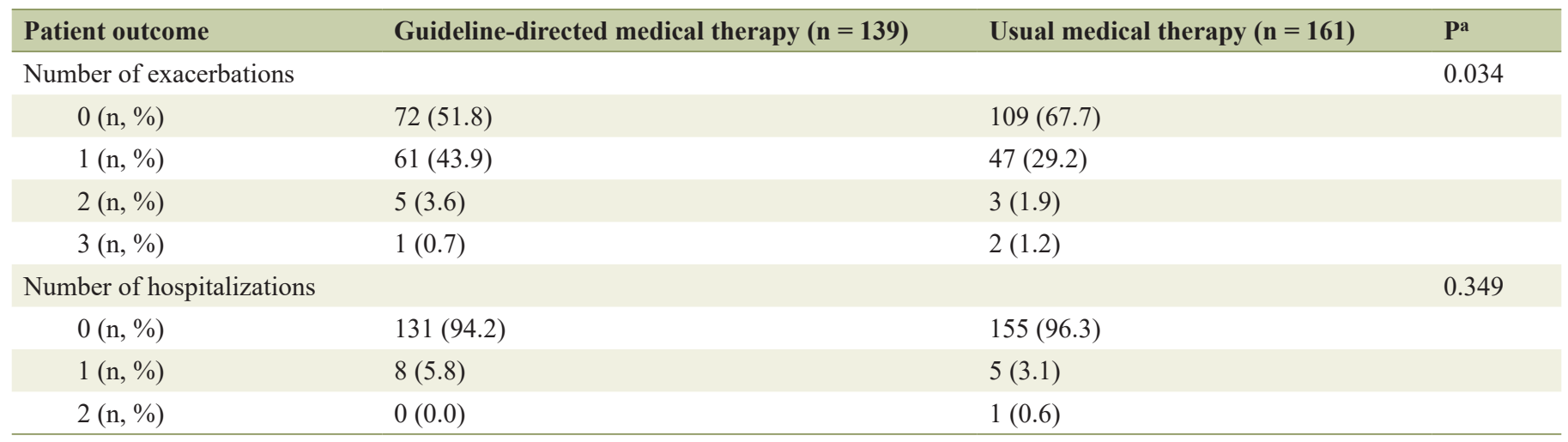

aa alndependent $t$-test.

erbations in the UMT group, with $29.2 \%$ suffering from one, $1.9 \%$ suffering from two, and $1.2 \%$ suffering from three $(\mathrm{P}<$ 0.05) (Table 5, Fig. 1). The number of recorded hospitalizations as a result of uncontrolled asthma exacerbations was also found to be higher in the GDMT group (5.8\% suffering from one in the last year) when compared to the UMT group $(3.1 \%$ suffering from one and $0.6 \%$ suffering two in the last year), although the difference was not found to be statistically significant $(\mathrm{P}=0.349)$ (Table 5).

\section{Discussion}

In our study, we found that nearly half of the patients in the primary care setting received guideline-directed therapy as per GINA [8]. Non-adherence to the GINA asthma management guidelines occurs due to a variety of reasons, including lack of knowledge of current guideline recommendations [14, 15], patient factors such as affordability or insurance coverage [16], and integration of guidelines within each clinic's EMRs [17, 18]. While physician awareness and patient factors do play a role in influencing the practice, guidelines being better integrated within an EMR, whether it be through decision support tools or best practice notifications, have also been shown to increase provider adherence to current recommendations [19]. There are no studies analyzing primary care providers' adher-

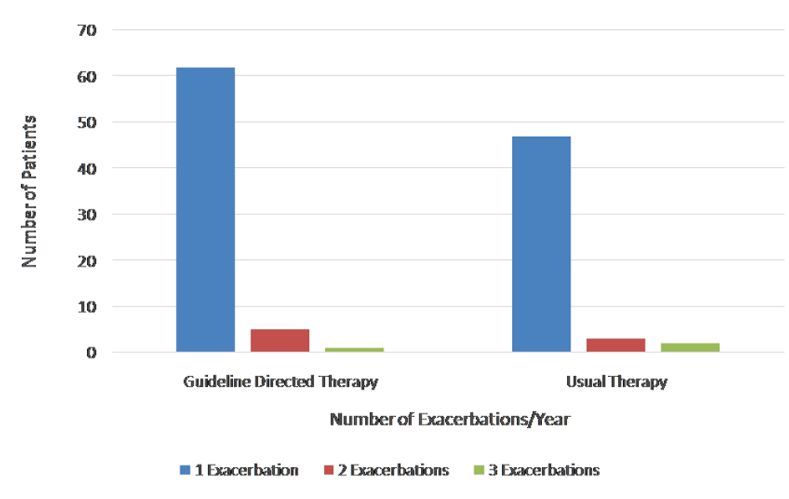

Figure 1. Frequencies of number of exacerbations per year. ence to the 2018 GINA guidelines and its impact on patient outcomes, hence our findings are unique.

In our study, we also found that there was no significant difference in the hospitalization rates for patients being treated with GDMT when compared to those receiving provider driven UMT. Interestingly, we found a significant decrease in the rate of exacerbations in the group of patients who received usual care when compared to those who received GDMT. Possible reasoning for this is that many patients who fell in the category of physician driven care were those who probably stepped up in therapy too soon, whether it be through addition of long-acting beta agonists prior to prescribing high-dose ICSs, or the addition of leukotriene inhibitors for patients with comorbid conditions, which the guidelines do not account for. These small variations done at the physician's discretion may have provided better control on a case-by-case basis when compared to the more generalized guidelines.

We also found that there were no significant differences in the outcomes between patients of different genders, race or BMI. There are studies that reported both a higher prevalence of disease $[20,21]$ and a higher rate of hospitalizations in patients of black or Hispanic race when compared to white; and less frequent office follow-up visits with the primary care providers by the patients of black and Hispanic races $[22,23]$. This is influenced by the finding that in the past patients of black or Hispanic race were less likely to be prescribed more advanced therapies, such as ICS [24, 25]. Lavoie et al found that elevated BMI was associated with poor asthma control, as measured by the patient subjective symptoms, but not severity [26]. Our findings are unique as we found no significant differences in the outcomes between patients of different genders, race or BMI.

Our findings also indicate that the patients suffering from congestive heart failure appear to have increased odds of suffering from exacerbations. Previously, this was thought to be due to beta blocker use and its effect on bronchospasm. Hanania et al found that patients carrying a diagnosis of mild bronchial asthma received increasing doses of nadolol with no findings of worsened symptoms or worsened pulmonary function testing [27]. Similarly, we found that the patients with allergic rhinitis had two times greater odds of having exacerbations. This is in line with the previous analyses showing an 
increased prevalence in emergency room visits [28] and difficulty achieving symptom control in patients suffering from asthma with diagnosed allergic rhinitis [29]. In addition, it has also been reported that treating the coexistent allergic rhinitis both improves asthma control and reduces health care burden $[30,31]$, whether it be through use of inhaled steroids or oral leukotriene inhibitors [30].

There were several limitations in this study. Being a retrospective review, we had a chance to examine patient records over the course of approximately 1 year only, which restricted our ability to predict longer term outcomes of patients on different asthma therapies. Additionally, the information obtained was through review of our EMR, and as such our ability to measure outcomes was dependent on diligent documentation at the time of the patients' visits.

The major strengths of our study were the review of clinical data of our established patients in our primary care office who were followed at a scheduled interval and were treated by the same care provider, which allowed us to analyze the provider specific management strategies, adherence and outcomes; as well as inclusion of a multitude of associated medical conditions that could have influenced the management outcomes.

\section{Conclusions}

We conclude that primary care providers' adherence to the 2018 GINA guidelines for asthma treatment did not offer benefit to patient outcomes, such as number of exacerbations or hospitalizations, compared to the usual medical care of bronchial asthma. Patient-tailored care may offer reduction in the rates of exacerbations and hospitalization.

\section{Acknowledgments}

The authors thank Christine Rickette RN (study coordinator) for her contribution to this study.

\section{Financial Disclosure}

None to declare.

\section{Conflict of Interest}

None to declare.

\section{Informed Consent}

Not applicable.

\section{Author Contributions}

JJ and SR made substantial contributions to the study design, drafting, data acquisition and analysis, and manuscript writing. $\mathrm{KH}$ analyzed the data. SR contributed in revising the manuscript critically for improved intellectual content, and final approval for the version to be published.

\section{Data Availability}

The authors declare that data supporting the findings of this study are available within the article.

\section{References}

1. Bousquet J, Dahl R, Khaltaev N. Global Alliance against Chronic Respiratory Diseases. Eur Respir J. 2007;29(2):233239.

2. Tanno LK, Haahtela T, Calderon MA, Cruz A, Demoly P, Joint Allergy A. Implementation gaps for asthma prevention and control. Respir Med. 2017;130:13-19.

3. Centers for Disease Control and Prevention. Asthma Data, Statistics, and Surveillance. 2020. Available at: https://www.cdc.gov/asthma/asthmadata.htm. Accessed on May 9, 2020.

4. Nurmagambetov T, Kuwahara R, Garbe P. The Economic Burden of Asthma in the United States, 2008-2013. Ann Am Thorac Soc. 2018;15(3):348-356.

5. Hanski I, von Hertzen L, Fyhrquist N, Koskinen K, Torppa K, Laatikainen T, Karisola P, et al. Environmental biodiversity, human microbiota, and allergy are interrelated. Proc Natl Acad Sci U S A. 2012;109(21):8334-8339.

6. van Tilburg Bernardes E, Arrieta MC. Hygiene Hypothesis in Asthma Development: Is Hygiene to Blame? Arch Med Res. 2017;48(8):717-726.

7. Horak F, Doberer D, Eber E, Horak E, Pohl W, Riedler J, Szepfalusi Z, et al. Diagnosis and management of asthma - Statement on the 2015 GINA Guidelines. Wien Klin Wochenschr. 2016;128(15-16):541-554.

8. Global initiative for asthma: a guide for health professionals updated 2018 based on the Global Strategy for Asthma Management and Prevention (GINA). 2018. Available at: https://ginasthma.org/wp-content/uploads/2019/01/2018GINA.pdf. Accessed on May 9, 2020.

9. Barnes PJ. Therapeutic approaches to asthma-chronic obstructive pulmonary disease overlap syndromes. J Allergy Clin Immunol. 2015;136(3):531-545.

10. Gionfriddo MR, Hagan JB, Rank MA. Why and how to step down chronic asthma drugs. BMJ. 2017;359:j4438.

11. Bai TR, Vonk JM, Postma DS, Boezen HM. Severe exacerbations predict excess lung function decline in asthma. Eur Respir J. 2007;30(3):452-456.

12. Ivanova JI, Bergman R, Birnbaum HG, Colice GL, Silverman RA, McLaurin K. Effect of asthma exacerbations on health care costs among asthmatic patients with moderate and severe persistent asthma. J Allergy Clin Immunol. 2012;129(5):1229-1235.

13. Miller MK, Lee JH, Miller DP, Wenzel SE, TENOR Study Group. Recent asthma exacerbations: a key predictor of future exacerbations. Respir Med. 2007;101(3):481-489. 
14. Goeman DP, Hogan CD, Aroni RA, Abramson MJ, Sawyer SM, Stewart K, Sanci LA, et al. Barriers to delivering asthma care: a qualitative study of general practitioners. Med J Aust. 2005;183(9):457-460.

15. Doerschug KC, Peterson MW, Dayton CS, Kline JN. Asthma guidelines: an assessment of physician understanding and practice. Am J Respir Crit Care Med. 1999;159(6):1735-1741.

16. Cooper V, Metcalf L, Versnel J, Upton J, Walker S, Horne R. Patient-reported side effects, concerns and adherence to corticosteroid treatment for asthma, and comparison with physician estimates of side-effect prevalence: a UKwide, cross-sectional study. NPJ Prim Care Respir Med. 2015;25:15026.

17. FitzGerald JM, Boulet LP, McIvor RA, Zimmerman S, Chapman KR. Asthma control in Canada remains suboptimal: the Reality of Asthma Control (TRAC) study. Can Respir J. 2006;13(5):253-259.

18. Gupta S, Rai N, Bhattacharrya O, Cheng AYY, Connelly KA, Boulet LP, Kaplan A, et al. Optimizing the language and format of guidelines to improve guideline uptake. CMAJ. 2016;188(14):E362-E368.

19. Cloutier MM. Asthma management programs for primary care providers: increasing adherence to asthma guidelines. Curr Opin Allergy Clin Immunol. 2016;16(2):142147.

20. Stanley AH, Demissie K, Rhoads GG. Asthma development with obesity exposure: observations from the cohort of the National Health and Nutrition Evaluation Survey Epidemiologic Follow-up Study (NHEFS). J Asthma. 2005;42(2):97-99.

21. Centers for Disease Control and Prevention. Asthma prevalence and control characteristics by race/ethnicity - United States, 2002. MMWR Morb Mortal Wkly Rep. 2004;53(7):145-148.

22. Murray MD, Stang P, Tierney WM. Health care use by inner-city patients with asthma. J Clin Epidemiol. 1997;50(2):167-174.

23. Grant EN, Lyttle CS, Weiss KB. The relation of socioeco- nomic factors and racial/ethnic differences in US asthma mortality. Am J Public Health. 2000;90(12):1923-1925.

24. Cydulka RK, Tamayo-Sarver JH, Wolf C, Herrick E, Gress S. Inadequate follow-up controller medications among patients with asthma who visit the emergency department. Ann Emerg Med. 2005;46(4):316-322.

25. Apter AJ, Boston RC, George M, Norfleet AL, Tenhave T, Coyne JC, Birck K, et al. Modifiable barriers to adherence to inhaled steroids among adults with asthma: it's not just black and white. J Allergy Clin Immunol. 2003;111(6):1219-1226.

26. Lavoie KL, Bacon SL, Labrecque M, Cartier A, Ditto B. Higher BMI is associated with worse asthma control and quality of life but not asthma severity. Respir Med. 2006;100(4):648-657.

27. Hanania NA, Singh S, El-Wali R, Flashner M, Franklin AE, Garner WJ, Dickey BF, et al. The safety and effects of the beta-blocker, nadolol, in mild asthma: an open-label pilot study. Pulm Pharmacol Ther. 2008;21(1):134-141.

28. Bousquet J, Gaugris S, Kocevar VS, Zhang Q, Yin DD, Polos PG, Bjermer L. Increased risk of asthma attacks and emergency visits among asthma patients with allergic rhinitis: a subgroup analysis of the investigation of montelukast as a partner agent for complementary therapy [corrected]. Clin Exp Allergy. 2005;35(6):723-727.

29. Valovirta E, Pawankar R. Survey on the impact of comorbid allergic rhinitis in patients with asthma. BMC Pulm Med. 2006;6(Suppl 1):S3.

30. Bousquet J, Schunemann HJ, Samolinski B, Demoly P, Baena-Cagnani CE, Bachert C, Bonini S, et al. Allergic Rhinitis and its Impact on Asthma (ARIA): achievements in 10 years and future needs. J Allergy Clin Immunol. 2012;130(5):1049-1062.

31. Dal Negro R, Piskorz P, Vives R, Guilera M, Sazonov Kocevar V, Badia X. Healthcare utilisation and costs associated with adding montelukast to current therapy in patients with mild to moderate asthma and co-morbid allergic rhinitis: PRAACTICAL study. Pharmacoeconomics. 2007;25(8):665-676. 